\title{
Odd-parity superconductivity in bilayer transition metal dichalcogenides
}

\author{
Yasuharu Nakamura ${ }^{1}$ and Youichi Yanase, \\ ${ }^{1}$ Graduate School of Science and Technology, Niigata University, Niigata 950-2181, Japan \\ ${ }^{2}$ Department of Physics, Graduate School of Science, Kyoto University, Kyoto 606-8502, Japan
}

(Dated: September 3, 2018)

\begin{abstract}
Spin-orbit coupling in transition metal dichalcogenides (TMDCs) causes spin-valley locking giving rise to unconventional optical, transport, and superconducting properties. In this paper, we propose exotic superconductivity in bilayer group-IV TMDCs by symmetry control. The sublatticedependent "hidden" spin-orbit coupling arising from local inversion symmetry breaking in the crystal structure may stabilize the odd-parity superconductivity by purely $s$-wave local pairing interaction. The stability of the odd-parity superconducting state depends on the bilayer stacking. The $2 \mathrm{H}_{b}$ stacking in $\mathrm{Mo}_{2}$ and $\mathrm{W}_{2}(X=\mathrm{S}, \mathrm{Se})$ favors the odd-parity superconductivity due to interlayer quantum interference. On the other hand, the odd-parity superconductivity is suppressed by the $2 \mathrm{H}_{a}$ stacking of $\mathrm{NbSe}_{2}$. Calculating the phase diagram of the tight-binding model derived from first principles band calculations, we conclude that the intercalated bilayer $\mathrm{MoS}_{2}$ and $\mathrm{WS}_{2}$ are candidates for a new class of odd-parity superconductors by spin-orbit coupling.
\end{abstract}

\section{INTRODUCTION}

The recent fabrication of atomically thin transition metal dichalcogenides (TMDCs) films has led to extraordinary developments in both applied and basic sciences $\underline{1-23}$. The discovery of superconductivity in $\mathrm{MoS}_{2} \underline{11}$ and related $\mathrm{TMDCs}^{20} \underline{\underline{23}}$ has demonstrated a new paradigm of artificial two-dimensional (2D) superconductors. Indeed, an exotic superconducting phase protected by spin-orbit coupling has been identified $17-19,23$.

The monolayer group-VI TMDCs have a hexagonal crystal structure sketched in Fig. 1(b), where the metal ions are surrounded by six chalcogen ligands forming a prism structure ${ }^{24}$. The point group symmetry is $D_{3 h}$, lacking the inversion symmetry. Thus, the monolayer TMDCs are intrinsically noncentrosymmetric. Therefore, the antisymmetric spin-orbit coupling (ASOC) appears and induces valley-dependent spin polarization along the crystallographic $c$-axis ${ }^{25}$. Since the two valleys around the $K$ and $K^{\prime}$ points in the Brillouin zone are time-reversal pairs, the ASOC induces such "spinvalley locking". The resulting spin-splitting of the band structure has been shown by first principles band structure calculations $\underline{24,26-32}$ and was observed in many experiments 24 .

A variety of intriguing phenomena caused by the spin-valley locking have been revealed by recent works. For instance, the superconducting state in the electrondoped $\mathrm{MoS}_{2}$ is protected against the paramagnetic depairing effect. As a consequence, a huge upper critical field above 50T, which significantly exceeds the PauliChandrasekhar-Clogston limit 33,34 , has been observed 17 . Then, the superconductivity is called "Ising superconductivity" $\underline{18,19}$. A peculiar optical response $e^{25,35}$ has also been observed $\stackrel{4-7}{-}$. Furthermore, topological superconductivity in TMDCs ${ }^{36}-38$ and TMDC-based devices $\underline{39}-\underline{42}$ has been theoretically proposed, and the possibility of the topological insulating phase was discussed $\stackrel{43}{ }$.

Tunability of van der Waals heterostructures enables symmetry control in TMDCs from monolayer to bulk.
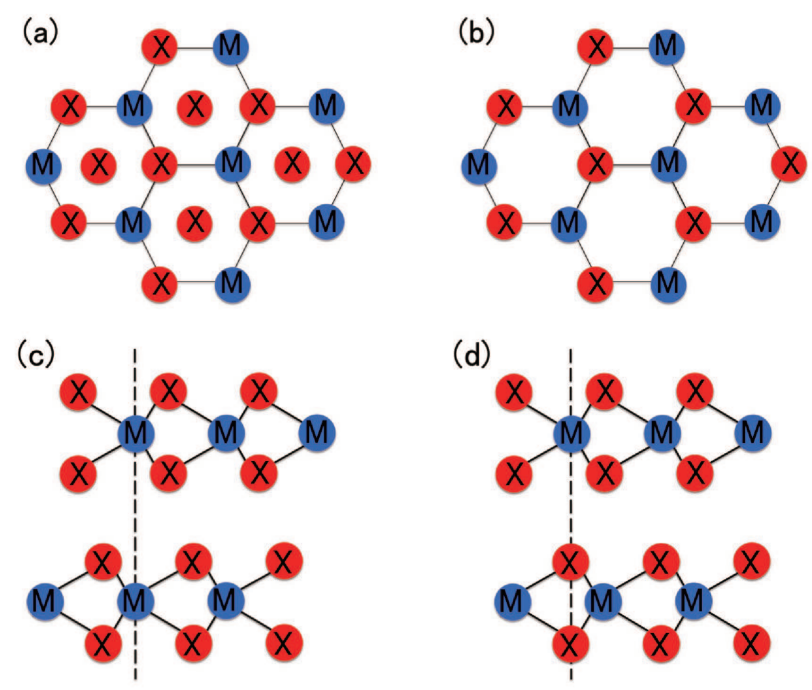

FIG. 1. (Color online) Crystal structures of bilayer TMDCs. Blue $\mathrm{M}$ and red $\mathrm{X}$ show the metal ions and chalcogen ligands, respectively. (a) and (c) are top-view. (b) and (d) are sideview. (a) and (b) illustrate $2 \mathrm{H}_{a}$ stacking structure, while (c) and $(\mathrm{d})$ show $2 \mathrm{H}_{b}$ stacking structure.

TABLE I. Point group symmetry of monolayer, bilayer, and bulk TMDCs having $2 \mathrm{H}_{b}$ structure.

$$
\begin{array}{l|c|c|c} 
& \text { monolayer } & \text { bilayer } & \text { bulk } \\
\hline \text { Point group } & D_{3 \mathrm{~h}} & D_{3 \mathrm{~d}} & D_{6 \mathrm{~h}}
\end{array}
$$

The chemically stable stacking structure is determined mainly by the metal ions 44 . For examples, the $2 \mathrm{H}_{b}$ structure is favored in group-VI $\mathrm{Mo}_{2}$ and $\mathrm{W} X_{2}(X=\mathrm{S}, \mathrm{Se})$, while the $2 \mathrm{H}_{a}$ structure is stable in group- $\mathrm{V} \mathrm{Nb} X_{2}$. The $2 \mathrm{H}_{a}$ and $2 \mathrm{H}_{b}$ stackings are sketched in Fig. 1. In both structures, the 2D coordinates of metal ions and chalcogen ligands are exchanged between the upper and lower 
layers. Therefore, the space inversion symmetry is recovered when the number of TMDC layers is even. For the $2 \mathrm{H}_{b}$ stacking, the point group is $D_{6 h}$ in the bulk, and $D_{3 d}$ in the bilayer, both of which preserve the space inversion symmetry. The symmetry of the heterostructures is summarized in Table I

Although the global inversion symmetry is preserved in bilayer TMDCs, the local site symmetry of metal ions is still noncentrosymmetric $D_{3 h}$. Such locally noncentrosymmetric crystal structure results in a sublatticedependent ASOC 45,46 . Although the spatial average of the ASOC disappears so as to preserve the global inversion symmetry, a sublattice-dependent spin polarization 45 has actually been observed in various materials $47-53$ including the TMDCs $50-52$. As unusual superconducting properties have been established in noncentrosymmetric systems ${ }^{54,55}$, it is naturally expected that unconventional superconducting states may be stabilized in locally noncentrosymmetric systems. Indeed, it has been shown that the "spin-momentum-layer locking" by the spin-orbit coupling may stabilize the odd-parity superconductivity in multilayer Rashba systems $\underline{56} \underline{\underline{60}}$ and a nonsymmorphic zigzag chain $\underline{61}$. Then, the spin-orbit coupling combined with the spin polarization causes the $\pi$-junction between the two sublattices and stabilizes the sign changing spin-singlet order parameter leading to odd-parity. Such a superconducting state has been identified as a pair-density-wave (PDW) state. Interestingly, multilayer odd-parity superconductivity is classified into the topological crystalline superconductivity $\underline{\underline{62}} \underline{\underline{64}}$, and zigzag chains are identified as $Z_{2}$ topological superconductors 61 . In this paper we propose the material realization of analogous odd-parity superconductivity in bilayer TMDCs.

We show advantages of bilayer TMDCs for realizing the odd-parity superconductivity without spin-triplet pairing. First, in the $2 \mathrm{H}_{b}$ structure, the interlayer hopping integral $t_{\perp} f(\boldsymbol{k})$ vanishes at the $K$ and $K^{\prime}$ point because of the quantum interference effect $\underline{24,65,66}$. Therefore, the ratio $\alpha / t_{\perp} f(\boldsymbol{k})$, with $\alpha$ being the coupling constant of ASOC, is enhanced on the Fermi surface. This ratio is increased further by intercalation, which induces the superconductivity in $\mathrm{K}_{x} \mathrm{MoS}_{2}, \mathrm{Rb}_{x} \mathrm{MoS}_{2}$, and $\mathrm{Cs}_{x} \mathrm{MoS}_{2}$ without gating 67 . The large $\alpha / t_{\perp} f(\boldsymbol{k})$ favors the oddparity superconductivity $\underline{58}$. On the other hand, in the $2 \mathrm{H}_{a}$ structure, a considerable interlayer hopping integral appears in the entire Brillouin zone, and therefore, we will see rather conventional behaviors in Nb-based TMDCs. Second, the magnetic field parallel to the conducting plane favors the odd-parity superconductivity while avoiding the orbital depairing effect, although the perpendicular field assumed in the multilayer Rashba systems $\underline{5-60}$ drastically suppresses the superconductivity. Fortunately, the Ising superconductivity in TMDCs is robust against the parallel magnetic field ${ }^{17}-\underline{19}$.

The 2D superconductors in parallel magnetic fields have been investigated in the context of the Fulde-FerrellLarkin-Ovchinnikov (FFLO) state $\underline{\underline{68}-71}$ and the helical superconducting state $\underline{\underline{54}, 55}$. However, we show that the odd-parity PDW state is more stable than the FFLO and helical states because of the peculiar symmetry of bilayer TMDCs.

This paper is organized as follows. In Sec. II, the model for bilayer TMDCs is introduced, and the mean field theory is explained. We show the main results in Secs. III and IV. In Sec. III, the superconducting phase diagram in the Pauli limit is shown. In Sec. IV, we calculate the phase diagram by taking into account both paramagnetic and orbital effects of the parallel magnetic field. It is shown that the odd-parity superconducting state, called the PDW state, is stable in intercalated bilayer $2 \mathrm{H}_{b}$-TMDCs. In Sec. V, the roles of the Rashbatype ASOC are examined. In Sec. VI, a brief summary is given, and characteristic properties of the odd-parity PDW state are discussed for a future experimental test.

\section{FORMULATION}

\section{A. Bilayer model}

We investigate superconductivity in bilayer TMDCs by taking intrinsic Zeeman-type ASOC into account. We focus on the electron-doped TMDCs in which superconductivity has been reported $11,17-22,67$, and we adopt a singleorbital tight-binding model for the transition metal $d_{z^{2-}}$ orbital24,

$$
\hat{H}=\hat{H}_{0}+\hat{H}_{\mathrm{I}}
$$

The single-particle Hamiltonian is composed of

$$
\hat{H}_{0}=\hat{H}_{\mathrm{k}}+\hat{H}_{\perp}+\hat{H}_{\mathrm{Z}}+\hat{H}_{\mathrm{R}}+\hat{H}_{\mathrm{P}},
$$

where

$$
\begin{aligned}
& \hat{H}_{\mathrm{k}}=\sum_{\mathbf{k}, m, s} \varepsilon\left(\mathbf{k}+\mathbf{p}_{m}\right) c_{\mathbf{k} m s}^{\dagger} c_{\mathbf{k} m s}, \\
& \hat{H}_{\perp}=t_{\perp} \sum_{\mathbf{k}, s} f_{\perp}(\mathbf{k}) c_{\mathbf{k} 1 s}^{\dagger} c_{\mathbf{k} 2 s}+h . c ., \\
& \hat{H}_{\mathrm{Z}}=\sum_{\mathbf{k}, m, s, s^{\prime}} \alpha_{\mathrm{Z}}^{(m)} \boldsymbol{g}_{\mathrm{Z}}\left(\mathbf{k}+\mathbf{p}_{m}\right) \cdot \boldsymbol{\sigma}_{s s^{\prime}} c_{\mathbf{k} m s}^{\dagger} c_{\mathbf{k} m s^{\prime}}, \\
& \hat{H}_{\mathrm{R}}=\sum_{\mathbf{k}, m, s, s^{\prime}} \alpha_{\mathrm{R}}^{(m)} \boldsymbol{g}_{\mathrm{R}}\left(\mathbf{k}+\mathbf{p}_{m}\right) \cdot \boldsymbol{\sigma}_{s s^{\prime}} c_{\mathbf{k} m s}^{\dagger} c_{\mathbf{k} m s^{\prime}}, \\
& \hat{H}_{\mathrm{P}}=-\frac{g \mu_{\mathrm{B}}}{2} \sum_{\mathbf{k}, m, s, s^{\prime}} \mathbf{H} \cdot \boldsymbol{\sigma}_{s s^{\prime}} c_{\mathbf{k} m s}^{\dagger} c_{\mathbf{k} m s^{\prime}},
\end{aligned}
$$

with $c_{\mathbf{k} m s}^{\dagger}$ being the creation operator for electrons with momentum $\mathbf{k}$ and spin $s$ on the $m$-th layer. The index for the layer takes $m=1,2$ in bilayer systems.

The first term is the kinetic energy term by hopping integrals in the $2 \mathrm{D}$ plane. Thus, we have

$$
\varepsilon(\mathbf{k})=2 t_{1}\left(\cos k_{\mathrm{y}} a+2 \cos \frac{\sqrt{3}}{2} k_{\mathrm{x}} a \cos \frac{1}{2} k_{\mathrm{y}} a\right)-\mu,
$$


by taking into account the nearest-neighbor hopping in the triangular lattice. The chemical potential $\mu$ is included in the dispersion relation $\varepsilon(\mathbf{k})$. We fix $2 \mathrm{D}$ carrier density per layer $n_{2 \mathrm{D}}=1 \times 10^{14} \mathrm{~cm}^{-2}$ throughout this paper. This carrier density is close to the optimal doping of superconducting $\mathrm{MoS}_{2} \frac{11}{1}$, and then, small Fermi surfaces enclose the $K$ and $K^{\prime}$ points in the Brillouin zone. Later, the carrier density dependence is discussed. The lattice constant is assumed to be $a=3.2 \AA$ in accordance with first principles calculations $\underline{72,73}$ and an experimental report $\underline{74}$. We choose the unit of energy $t_{1}=1$, which is estimated to be $t_{1} \simeq 200 \mathrm{meV}^{75}$.

The second term $\hat{H}_{\perp}$ is the interlayer hopping energy which depends on the stacking structure. The interlayer hybridyzation function is

$$
f_{\perp}(\mathbf{k})=1,
$$

for the $2 \mathrm{H}_{a}$ structure, while it is

$$
\begin{aligned}
f_{\perp}(\mathbf{k}) & =\frac{1}{3}\left[\cos \frac{k_{\mathrm{x}}}{\sqrt{3}} a+i \sin \frac{k_{\mathrm{x}}}{\sqrt{3}} a\right. \\
& \left.+2\left(\cos \frac{k_{\mathrm{x}}}{2 \sqrt{3}} a-i \sin \frac{k_{\mathrm{x}}}{2 \sqrt{3}} a\right) \cos \frac{1}{2} k_{\mathrm{y}} a\right],
\end{aligned}
$$

for the $2 \mathrm{H}_{b}$ structure. For non-intercalated TMDCs, we assume $t_{\perp} / t_{1}=0.6$ in accordance with the band structure calculation for the bilayer $\mathrm{MoS}_{2} \frac{72}{}$. However, much smaller $t_{\perp}$ is adopted for intercalated TMDCs, since it has been shown that the interlayer hopping is significantly decreased by the intercalation $\underline{\underline{76}}$.

The third and fourth terms represent ASOCs. The structure of ASOC has been classified by group theory 77 . Although the Rashba-type ASOC in polar point groups has been studied intensively $\stackrel{54}{\underline{4}}$, other kinds of ASOC may appear in non-polar point groups. Indeed, 21 point groups out of a total of 32 are noncentrosymmetric. Interestingly, the 2D materials classified into the $D_{3 \mathrm{~h}}$ point group show a uniaxial ASOC, which causes spin polarization along the crystallographic $c$-axis. Such ASOC called "Zeeman-type" ASOC 17 is represented by $\hat{H}_{\mathrm{Z}}$, in which the spin texture in the momentum space is given by

$$
\boldsymbol{g}_{\mathrm{Z}}(\mathbf{k})=\frac{2}{3 \sqrt{3}}\left(0,0, \sin k_{\mathrm{y}}-2 \cos \frac{\sqrt{3}}{2} k_{\mathrm{x}} \sin \frac{1}{2} k_{\mathrm{y}}\right) .
$$

This term arises from the intrinsic (local) inversion symmetry breaking in the crystal structure of trigonal prismatic TMDCs. We also take into account the Rashbatype ASOC term $\hat{H}_{\mathrm{R}}$, which comes from the bilayer structure $\underline{45}$. Taking into account the nearest-neighbour coupling, we have the g-vector

$$
\begin{gathered}
\boldsymbol{g}_{\mathrm{R}}(\mathbf{k})=\frac{1}{1.7602}\left(-\sin k_{\mathrm{y}}-\cos \frac{\sqrt{3}}{2} k_{\mathrm{x}} \sin \frac{1}{2} k_{\mathrm{y}},\right. \\
\left.\sqrt{3} \sin \frac{\sqrt{3}}{2} k_{\mathrm{x}} \cos \frac{1}{2} k_{\mathrm{y}}, 0\right) .
\end{gathered}
$$

The constant factors are chosen so that the maximum amplitude is unity, that is, $\operatorname{Max}_{\mathbf{k}}\left|f_{\perp}(\mathbf{k})\right|=$
$\operatorname{Max}_{\mathbf{k}}\left|\boldsymbol{g}_{\mathrm{Z}}(\mathbf{k})\right|=\operatorname{Max}_{\mathbf{k}}\left|\boldsymbol{g}_{\mathrm{R}}(\mathbf{k})\right|=1$. Because the global inversion symmetry is recovered by the bilayer stacking, the layer-dependent coupling constants change sign, $\left(\alpha_{\mathrm{Z}}^{(1)}, \alpha_{\mathrm{Z}}^{(2)}\right)=\left(\alpha_{\mathrm{Z}},-\alpha_{\mathrm{Z}}\right)$ and $\left(\alpha_{\mathrm{R}}^{(1)}, \alpha_{\mathrm{R}}^{(2)}\right)=\left(\alpha_{\mathrm{R}},-\alpha_{\mathrm{R}}\right)$, and thus the spatial averages vanish. This is the sublattice-dependent ASOC which is characteristic of locally noncentrosymmetric systems $\stackrel{45,46}{ }$. A coupling constant of Zeeman-type ASOC, $\alpha_{\mathrm{Z}} / t_{1}=0.0375$, is adopted unless mentioned otherwise. Then, the spin splitting energy on the Fermi surface is $2 \alpha_{\mathrm{Z}}\left|\boldsymbol{g}_{\mathrm{Z}}\left(\mathbf{k}_{\mathrm{F}}\right)\right| \simeq 13 \mathrm{meV}$ in accordance with the first principles band structure calculation for $\mathrm{MoS}_{2} \frac{17}{}$. The band structure calculation has also shown that the Rashba-type ASOC is much smaller than the Zeeman-type one $\underline{17}$. Thus, we set $\alpha_{\mathrm{R}}=0$ except in Sec. V.

In this paper, we study superconducting states in a parallel magnetic field, whose effects appear in two ways. One of the effects of the magnetic field is the paramagnetic effect, which is represented by the Zeeman coupling term $\hat{H}_{\mathrm{P}}$. This term plays an essential role in this work. For simplicity, we adopt the g-factor $g=2$ and fix the direction of the magnetic field along the [100]-axis. The other effect is the orbital effect taken into account through the Peierls phase. When the magnetic field is parallel to the $2 \mathrm{D}$ plane, the Peierls phase leads to a shift in momentum $\mathbf{k} \rightarrow \mathbf{k}+\frac{e}{\hbar} \mathbf{A}$. For the magnetic field along the [100]-axis, we can choose the vector potential $\mathbf{A}=-H z \hat{y}$. Then, Eqs. (3), (5), and (6) are modified by $\mathbf{p}_{m}=(3 / 2-m) \frac{e}{\hbar} H c \hat{y}$ with $c$ being the lattice constant along the $c$-axis. We set $c=6.15 \AA$ for non-intercalated TMDCs $88-80$ and assume $c=1.5 \times 6.15 \AA=9.225 \AA$ for intercalated TMDCs $\frac{67}{}$. Thus, the orbital effect is enhanced by intercalation.

Many previous works have theoretically studied superconducting states in the paramagnetic field. It has been shown that the FFLO state is stable in a high magnetic field of centrosymmetric systems $\stackrel{70,71}{ }$, while the helical state is stabilized in a low magnetic field of noncentrosymmetric systems ${ }^{54,55}$. Contrary to those studies, we show that the odd-parity PDW state may be more stable in bilayer TMDCs than the FFLO and helical states because of the local noncentrosymmetricity in the crystal structure.

In order to study superconductivity emerging from the peculiar electronic state, a momentum-independent $s$ wave pairing interaction is introduced,

$$
\hat{H}_{\mathrm{I}}=-V \sum_{i, m} n_{i m \uparrow} n_{i m \downarrow}
$$

with $n_{\text {ims }}$ being the number density operator for spin $s$ at the site $i$ on the layer $m$. Although the Zeemantype and Rashba-type ASOCs induce spin-triplet $p$-wave and $f$-wave components in the order parameter, respectively, we assume a purely $s$-wave pairing interaction for simplicity. This is justified because the effects of small spin-triplet components on the superconducting phase diagram are negligible ${ }^{\underline{59}}$. We do not address the possibility of non-s-wave superconductivity by strong elec- 
tron correlations $36,38,81,82$. First principles calculations for the $s$-wave superconductivity by electron-phonon interactions ${ }^{83}-85$ reproduce the observed superconducting dome ${ }^{11}$, supporting our assumption. We fix the transition temperature of superconductivity at zero magnetic field, $T_{\mathrm{c} 0}=5 \mathrm{~K}$, by tuning the coupling constant $V$.

\section{B. Mean field theory}

We analyze the model by means of the mean field theory. When the superconducting state is spatially uniform in the 2D plane, the layer-dependent order parameter $\Delta_{m}=V \sum\left\langle c_{\mathbf{k} m \uparrow} c_{-\mathbf{k} m \downarrow}\right\rangle$ is calculated by decoupling the interaction term,

$$
\hat{H}_{\mathrm{I}} \rightarrow \sum_{\mathbf{k}, m}\left(\Delta_{m} c_{\mathbf{k} m \uparrow}^{\dagger} c_{-\mathbf{k} m \downarrow}^{\dagger}+\text { h.c. }\right)+\sum_{m} \frac{\left|\Delta_{m}\right|^{2}}{V} .
$$

Then, the Bogoliubov-de Gennes (BdG) Hamiltonian is diagonalized by transforming the basis

$$
\begin{aligned}
\hat{C}^{\dagger} & =\left(c_{\mathbf{k} 1 \uparrow}^{\dagger}, c_{\mathbf{k} 1 \downarrow}^{\dagger}, \cdots, c_{-\mathbf{k} 2 \uparrow}, c_{-\mathbf{k} 2 \downarrow}\right) \\
& \rightarrow\left(\hat{\gamma}_{1 \mathbf{k}}^{\dagger}, \cdots, \hat{\gamma}_{8 \mathbf{k}}^{\dagger}\right)=\hat{C}^{\dagger} U(\mathbf{k}),
\end{aligned}
$$

with the unitary matrix,

$$
U(\mathbf{k})=\left(\begin{array}{ccc}
u_{1 \uparrow 1}(\mathbf{k}) & \cdots & u_{1 \uparrow 8}(\mathbf{k}) \\
\ddots & \vdots & \ddots \\
v_{2 \downarrow 1}(\mathbf{k}) & \cdots & v_{2 \downarrow 8}(\mathbf{k})
\end{array}\right)
$$

The stationary solution for the layer-dependent order parameter is obtained by the self-consistent equation,

$$
\Delta_{m}=-V \sum_{\mathbf{k}, \nu} u_{m \uparrow \nu}(\mathbf{k}) v_{m \downarrow \nu}^{*}(\mathbf{k}) f\left[E_{\nu}(\mathbf{k})\right],
$$

where $E_{\nu}(\mathbf{k})$ is the $\nu$-th eigenenergy at the momentum k. The free energy is obtained by calculating

$$
\begin{aligned}
F= & \frac{1}{2} \sum_{\mathbf{k}, \nu} E_{\nu}(\mathbf{k}) f\left[E_{\nu}(\mathbf{k})\right]+\frac{1}{2} T \sum_{\mathbf{k}, \nu} f\left[E_{\nu}(\mathbf{k})\right] \ln f\left[E_{\nu}(\mathbf{k})\right] \\
& +\sum_{m} \frac{\left|\Delta_{m}\right|^{2}}{V} .
\end{aligned}
$$

The thermodynamically stable state is determined by comparing the free energy of metastable states. The BCS state corresponds to the solution $\Delta_{1}=\Delta_{2}$, and the PDW state is characterized by the sign changing order parameter, $\Delta_{1}=-\Delta_{2}$. The odd-parity superconductivity is realized in the PDW state by the sublattice degree of freedom, without requiring the spin-triplet pairing. We confirmed that the metastable solutions are spatially uniform in the absence of the orbital effect and Rashba-type ASOC. In Secs. IV and V, we discuss the roles of the orbital effect and the Rashba-type ASOC, respectively.
However, in the main part of this paper (Sec. III), these minor effects are neglected.

The numerical calculation for spatially non-uniform states requires a long computational time owing to long coherence length resulting from the small energy scale of superconductivity, $T_{\mathrm{c} 0} \simeq 5 \mathrm{~K}$. Thus, we solve the linearized BdG equation instead of solving the full BdG equation, when we study the non-uniform superconducting state in Secs. IV and V. Superconducting states near the second order critical point are elucidated by the linearized theory capturing the divergence of superconducting susceptibility, $\hat{\chi}_{m m^{\prime}}(\mathbf{q})$. Adopting the T-matrix approximation, we obtain

$$
\hat{\chi}(\mathbf{q})=\frac{\hat{\chi}^{0}(\mathbf{q})}{\hat{1}-V \hat{\chi}^{0}(\mathbf{q})}
$$

where the irreducible susceptibility is calculated by

$$
\begin{aligned}
\hat{\chi}_{m m^{\prime}}^{0}(\mathbf{q}) & = \\
k_{\mathrm{B}} T \sum_{\mathbf{k}, l} & {\left[G_{m m^{\prime}}^{\uparrow \uparrow}\left(\mathbf{q} / \mathbf{2}+\mathbf{k}, i \omega_{l}\right) G_{m m^{\prime}}^{\downarrow \downarrow}\left(\mathbf{q} / \mathbf{2}-\mathbf{k},-i \omega_{l}\right)\right.} \\
& \left.\quad-G_{m m^{\prime}}^{\uparrow \downarrow}\left(\mathbf{q} / \mathbf{2}+\mathbf{k}, i \omega_{l}\right) G_{m m^{\prime}}^{\uparrow \downarrow}\left(\mathbf{q} / \mathbf{2}-\mathbf{k},-i \omega_{l}\right)\right] .
\end{aligned}
$$

$G_{m m^{\prime}}^{s s^{\prime}}\left(\mathbf{k}, i \omega_{l}\right)$ is the Green function on the Matsubara frequency, $\omega_{l}=(2 l+1) \pi k_{\mathrm{B}} T$. The superconducting instability occurs when the maximum eigenvalue of $V \hat{\chi}^{0}(\mathbf{q})$ is unity. The eigenvector is proportional to $\Delta_{m}(\mathbf{q})=$ $V \sum\left\langle c_{\mathbf{q} / \mathbf{2}+\mathbf{k} m \uparrow} c_{\mathbf{q} / \mathbf{2}-\mathbf{k} m \downarrow}\right\rangle$.

TABLE II. Classification of superconducting states. Uniform BCS and PDW states as well as non-uniform FFLO, helical, Josephson vortex, and CS states are specified by the spatial dependence of order parameter (right column).

\begin{tabular}{c|c|c} 
Uniform & $\operatorname{BCS}\left(A_{1 \mathrm{~g}}\right)$ & $\Delta_{1}(\mathbf{r})=\Delta_{2}(\mathbf{r})=\Delta$ \\
\cline { 2 - 3 }$(\mathbf{q}=0)$ & $\operatorname{PDW}\left(A_{2 \mathbf{u}}\right)$ & $\Delta_{1}(\mathbf{r})=-\Delta_{2}(\mathbf{r})=\Delta$ \\
\hline & $\mathrm{LO}$ & $\Delta_{1}(\mathbf{r})=\Delta_{2}(\mathbf{r})=\Delta \cos (\mathbf{q} \cdot \mathbf{r})$ \\
\cline { 2 - 3 } & $\mathrm{FF} /$ helical & $\Delta_{1}(\mathbf{r})=\Delta_{2}(\mathbf{r})=\Delta e^{i \mathbf{q} \cdot \mathbf{r}}$ \\
\cline { 2 - 3 } Non-Uniform & Josephson vortex & $\Delta_{1}(\mathbf{r})=\Delta\left(e^{-i \mathbf{q} \cdot \mathbf{r}}+\delta e^{i \mathbf{q} \cdot \mathbf{r}}\right)$ \\
$(\mathbf{q} \neq 0)$ & & $\Delta_{2}(\mathbf{r})=\Delta\left(\delta e^{-i \mathbf{q} \cdot \mathbf{r}}+e^{i \mathbf{q} \cdot \mathbf{r}}\right)$ \\
\cline { 2 - 3 } & $\mathrm{CS}$ & $\Delta_{1}(\mathbf{r})=\Delta\left(e^{i \mathbf{q} \cdot \mathbf{r}}+\delta e^{-i \mathbf{q} \cdot \mathbf{r}}\right)$ \\
& & $\Delta_{2}(\mathbf{r})=\Delta\left(\delta e^{i \mathbf{q} \cdot \mathbf{r}}+e^{-i \mathbf{q} \cdot \mathbf{r}}\right)$
\end{tabular}

The classification of superconducting states is summarized in Table II] The uniform superconducting states are BCS and PDW states. The concept of PDW state was introduced for spatially inhomogeneous superconducting states in the atomic length scale which is much shorter than the coherence length ${ }^{86}$. In the subsequent works ${ }^{5-64}$, however, the sign changing order parameter between sublattices has also been classified into the PDW state. In the latter case, the translation symmetry is not 
broken $(\mathbf{q}=0)$. On the other hand, the irreducible representation of order parameter differs from the BCS state. While the BCS state belongs to the $A_{1 \mathrm{~g}}$ irreducible representation, the PDW state belongs to the odd-parity $A_{2 \mathrm{u}}$ irreducible representation of $D_{3 \mathrm{~d}}$ point group.

When the center-of-mass momentum $\mathbf{q}$ is finite, the translation symmetry is broken. Then, the superconducting state may be classified into the FFLO $\underline{68} \underline{\underline{71}}$, helical ${ }^{54,55}$, complex-stripe $(\mathrm{CS})^{87}$, or Josephson vortex states. The detailed definition of these non-uniform states has been given in Ref. 63. In the case studied here, the Josephson vortex state (Secs. IV and V) and CS state (Sec. V) may be stable. Both states are regarded as an intermediate state between the Fulde-Ferrell (FF) state 6 a and the Larkin-Ovchinnikov (LO) state ${ }^{69}$. The order parameter is a superposition of $\Delta_{m}(\mathbf{q})$ and $\Delta_{m}(-\mathbf{q})$. Then, both amplitude and phase of order parameter are spatially non-uniform $\frac{87}{7}$, although only the amplitude (phase) is non-uniform in the LO (FF) state.

\section{PHASE DIAGRAM IN THE PAULI LIMIT}

First, we examine the superconducting phase diagram in the Pauli limit by setting $\mathbf{p}_{m}=0$. Figure 2 shows the $H$-T phase diagram of $2 \mathrm{H}_{b}$ structure. For small interlayer hopping integrals, $t_{\perp} / t_{1}=0.06$ and $t_{\perp} / t_{1}=0.125$, the odd-parity PDW state is stable in the high magnetic field region [Figs. 2(a) and (b)]. Although the PDW state is not stable for a moderate interlayer hopping, $t_{\perp} / t_{1}=0.6$, consistent with non-intercalated $\mathrm{MoS}_{2}\left[\right.$ Fig. $[2(\mathrm{c})], t_{\perp} / t_{1}$ is decreased in the intercalated TMDCs $\frac{76}{6}$, suggested here as a candidate of odd-parity superconductors.

The $2 \mathrm{H}_{b}$ stacking structure plays an important role in stabilizing the PDW state. Indeed, the $2 \mathrm{H}_{a}$ structure realizes only the conventional BCS state [Fig. 2(d)] even when the interlayer hopping is small. The distinct difference between the $2 \mathrm{H}_{a}$ and $2 \mathrm{H}_{b}$ structures comes from the interlayer hybridyzation function, $f_{\perp}(\mathbf{k})$. Although it is momentum-independent in the $2 \mathrm{H}_{a}$ structure, the $f_{\perp}(\mathbf{k})$ disappears at the $K$ and $K^{\prime}$ points in the $2 H_{b}$ structure because of the quantum interference of three interlayer hopping integrals $24,65,66$. Figure 3 plots the momentum dependence of the interlayer hybridyzation function, (10), as well as the magnitude of the Zeemantype g-vector, (11). We see that $f_{\perp}(\mathbf{K})=f_{\perp}\left(\mathbf{K}^{\prime}\right)=0$ while the g-vector $\boldsymbol{g}_{\mathrm{Z}}(\mathbf{k})$ takes the maximum amplitude at $\mathbf{k}=\mathbf{K}$ and $\mathbf{K}^{\prime}$. Therefore, the ratio of the ASOC and interlayer hybridyzation diverges at the $K$ point,

$$
\alpha_{\mathrm{Z}}\left|\boldsymbol{g}_{\mathrm{Z}}(\mathbf{K})\right| / t_{\perp}\left|f_{\perp}(\mathbf{K})\right|=\infty .
$$

Since the Fermi momentum is in the vicinity of the $K$ or $K^{\prime}$ point, the ratio may be large,

$$
\alpha_{\mathrm{Z}}\left|\boldsymbol{g}_{\mathrm{Z}}\left(\mathbf{k}_{\mathrm{F}}\right)\right| / t_{\perp}\left|f_{\perp}\left(\mathbf{k}_{\mathrm{F}}\right)\right|>1,
$$

on the Fermi surface even when $\alpha_{\mathrm{Z}} / t_{\perp} \ll 1$. The disappearance of the interlayer hybridyzation is protected
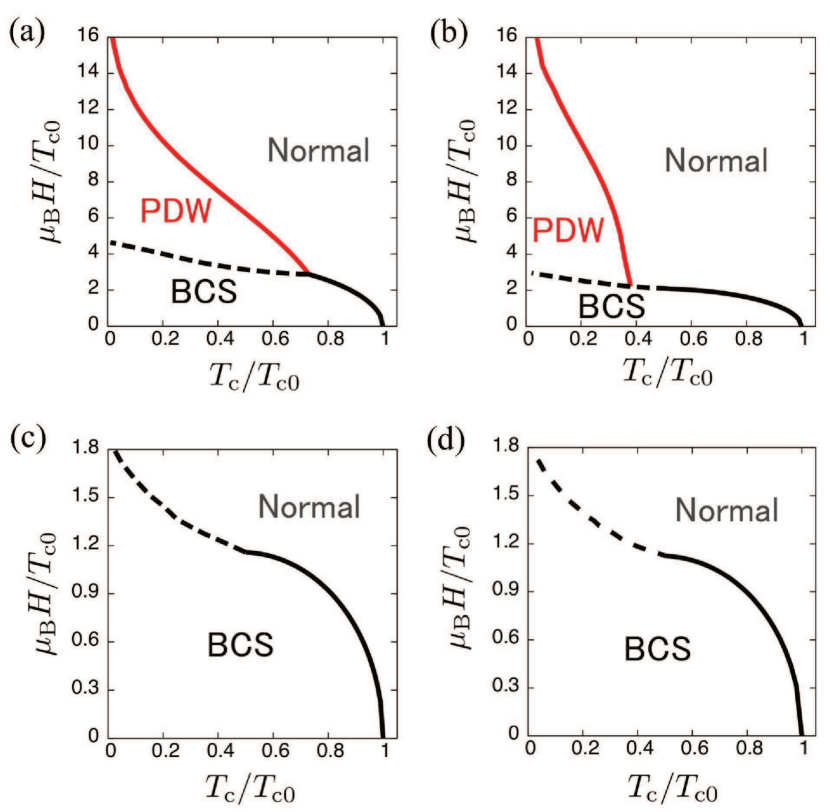

FIG. 2. (Color online) Superconducting phase diagram in the Pauli limit. (a-c) $2 \mathrm{H}_{b}$ stacking structure with (a) $t_{\perp} / t_{1}=$ 0.06 , (b) $t_{\perp} / t_{1}=0.125$, and (c) $t_{\perp} / t_{1}=0.6$. (d) $2 \mathrm{H}_{a}$ stacking structure with $t_{\perp} / t_{1}=0.125$. Solid (dashed) line shows the second order (first order) transition. The PDW state may be stable in the high magnetic field region of the $2 \mathrm{H}_{b}$ structure, although the BCS state is stable in the whole superconducting phase of the $2 \mathrm{H}_{a}$ structure. We assume $\alpha_{\mathrm{Z}}=0.0375$ in accordance with $\mathrm{MoS}_{2}$.

by the 3 -fold rotation symmetries $24,65,66$, and thus above features are not an artifact of our model but general properties of the $2 \mathrm{H}_{b}$ structure without orbital degeneracy. (a)

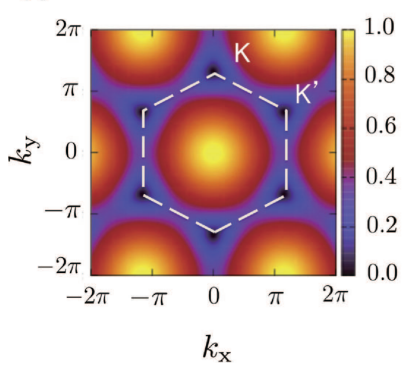

(b)

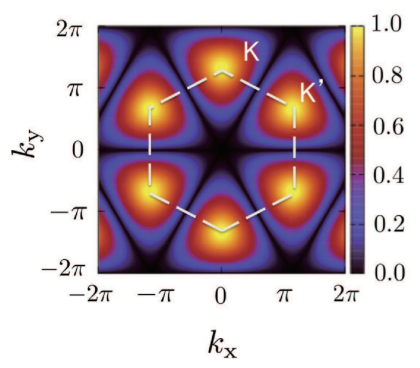

FIG. 3. (Color online) Momentum dependence of (a) the interlayer hybridyzation function $\left|f_{\perp}(\mathbf{k})\right|$ for the $2 \mathrm{H}_{b}$ structure and (b) the magnitude of Zeeman-type ASOC, $\left|\boldsymbol{g}_{\mathrm{Z}}(\mathbf{k})\right|$. Hexagonal white dashed line shows the Brillouin zone boundary, whose vertex is the $K$ and $K^{\prime}$ points.

Previous studies have shown that the sublatticedependent ASOC plays an important role when the ratio $\alpha_{\mathrm{Z}}\left|\boldsymbol{g}_{\mathrm{Z}}\left(\mathbf{k}_{\mathrm{F}}\right)\right| / t_{\perp}\left|f_{\perp}\left(\mathbf{k}_{\mathrm{F}}\right)\right|$ is large $\frac{45}{5}$. For the PDW state to be stabilized, the condition $\alpha_{\mathrm{Z}}\left|\boldsymbol{g}_{\mathrm{Z}}\left(\mathbf{k}_{\mathrm{F}}\right)\right| / t_{\perp}\left|f_{\perp}\left(\mathbf{k}_{\mathrm{F}}\right)\right| \geq 1$ 
has to be satisfied 58 . Thus, the relation (22) indicates the stable PDW state in the $2 \mathrm{H}_{b}$ structure. For the parameters $\alpha_{\mathrm{Z}}=0.0375, t_{\perp}=0.125$ and $n_{2 \mathrm{D}}=1 \times 10^{14}$ $\mathrm{cm}^{-2}$, the ratio is $\alpha_{\mathrm{Z}}\left|\boldsymbol{g}_{\mathrm{Z}}\left(\mathbf{k}_{\mathrm{F}}\right)\right| / t_{\perp}\left|f_{\perp}\left(\mathbf{k}_{\mathrm{F}}\right)\right|=1.68>1$, satisfying the condition. A smaller carrier density makes Fermi momentum to be closer to the $K$ point, and then the ratio $\alpha_{\mathrm{Z}}\left|\boldsymbol{g}_{\mathrm{Z}}\left(\mathbf{k}_{\mathrm{F}}\right)\right| / t_{\perp}\left|f_{\perp}\left(\mathbf{k}_{\mathrm{F}}\right)\right|$ is increased. Therefore, the PDW state is furthermore stable in the low carrier density region.

On the other hand, the condition for the thermodynamically stable PDW state is approximately reduced to $\alpha_{\mathrm{Z}} / t_{\perp} \geq 1$ in the $2 \mathrm{H}_{a}$ structure since $f_{\perp}(\mathbf{k})=1$. This condition is hardly satisfied in $\mathrm{NbSe}_{2}$ having a moderate spin-orbit coupling. Therefore, the odd-parity superconductivity is unlikely in the bilayer $2 \mathrm{H}_{a}-\mathrm{NbSe}_{2} \underline{\underline{19}}$.
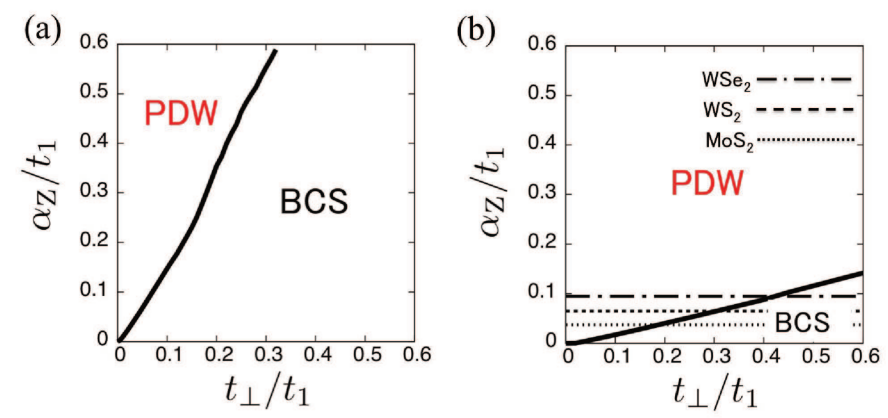

FIG. 4. (Color online) Phase diagram as a function of $\alpha_{Z}$ and $t_{\perp}$ for (a) the $2 \mathrm{H}_{a}$ structure and (b) the $2 \mathrm{H}_{b}$ structure. The temperature is fixed to be $T / T_{\mathrm{c} 0}=0.1$ and the magnetic field is tuned so as to be just below the upper critical field. In (b) the dotted, dashed, and dot-dashed lines show $\alpha_{\mathrm{Z}} / t_{1}$ in $\mathrm{MoS}_{2}, \mathrm{WS}_{2}$, and $\mathrm{WSe}_{2}$, respectively.

The contrasting behavior of the $2 \mathrm{H}_{a}$ and $2 \mathrm{H}_{b}$ structures is illuminated by Fig. 4 , which shows the phase diagram in the $\alpha_{\mathrm{Z}}-t_{\perp}$ plane at a low temperature $T / T_{\mathrm{c} 0}=$ 0.1 . In the $2 \mathrm{H}_{a}$ structure, the PDW state is stable only when $\alpha_{\mathrm{Z}} / t_{\perp} \geq 2$. On the other hand, the condition for the PDW state is significantly relaxed in the $2 \mathrm{H}_{b}$ structure to $\alpha_{\mathrm{Z}} / t_{\perp} \geq 0.25$.

\section{ORBITAL EFFECT}

Next, we examine the orbital effect on the superconducting phases. Although the orbital effect is completely suppressed in the monolayer, it may affect bilayer TMDCs. Since we consider the magnetic field parallel to the 2D plane, the Abrikosov vortex lattice state is not realized. However, the orbital effect may induce the Josephson vortex which penetrates into the atomic bilayers.

The Josephson vortex state is characterized by an order parameter with finite center-of-mass momentum $\mathbf{q}^{63}$, and thus it is distinguished from the PDW state as well as from the BCS state. We calculate the upper critical field for various center-of-mass momentum q of Cooper pairs by solving the linearized BdG equation. The superconducting state with the highest upper critical field is stable near the critical point.

Figures 5(a) and (b) show the upper critical fields of the BCS, PDW, and Josephson vortex states for the same parameters as Fig. 2(b). The increase in the $c$-axis lattice constant by intercalation $\frac{67}{}$ is taken into account in Fig. 5(b), although it is neglected in Fig. [5(a). Both figures show that the PDW state in the $2 \mathrm{H}_{b}$ structure is robust against the orbital effect at low temperatures although the Josephson vortex state is stable in the intermediate temperature region.

On the other hand, we find that the PDW state is suppressed when the Zeeman-type ASOC is furthermore increased. Although the parameters compatible with intercalated $\mathrm{MoS}_{2}$ have been assumed in Fig. 5(b), the ASOC is increased in Figs. 5(d) and (f) while maintaining the other parameters. The coupling constants $\alpha_{\mathrm{Z}}=0.065$ and $\alpha_{\mathrm{Z}}=0.095$ are consistent with the spin splitting energy at the $K$ point $\sim 26 \mathrm{meV}$ and $\sim 38 \mathrm{meV}$ in $\mathrm{WS}_{2}$ and $\mathrm{WSe}_{2}$, respectively. Then, we obtain the ratio, $\alpha_{\mathrm{Z}}\left|\boldsymbol{g}_{\mathrm{Z}}\left(\mathbf{k}_{\mathrm{F}}\right)\right| / t_{\perp} f_{\perp}\left(\mathbf{k}_{\mathrm{F}}\right)=2.91$ and 4.25 , respectively. As the Zeeman-type ASOC is increased, the upper critical field of the Josephson vortex state is enhanced although that of the PDW state is insensitive to the ASOC. Therefore, the PDW state becomes less stable than the Josephson vortex state. For a large ASOC compatible with $\mathrm{WSe}_{2}$, the PDW state is completely suppressed [Fig. 5(f)]. Thus, we conclude that the intercalated bilayer $\mathrm{MoS}_{2}$ is the best platform for the odd-parity PDW state rather than the heavier TMDCs.

The ASOC dependence is understood by paying attention to the paramagnetic depairing effect. The PDW state completely avoids the paramagnetic depairing effect, because the symmetry of superconductivity is the same as spin triplet superconductivity $\underline{45}$. On the other hand, the BCS and Josephson vortex states are suppressed by the paramagnetic depairing effect. This is indeed the reason why the PDW state is stable in the high magnetic field region ${ }^{58}$. However, the Zeeman-type ASOC protects the BCS and Josephson vortex states against the paramagnetic depairing effect. Hence, the upper critical field of the Josephson vortex state increases by increasing the ASOC. These features have been demonstrated in Fig. 5. The Josephson vortex state has also been demonstrated in a recent study for bilayer TMDCs 88 , although the PDW state has not been shown. Combining with the results in the paramagnetic limit (Sec. III), we find that an intermediate value of the Zeeman-type ASOC satisfying,

$$
1 \leq \alpha_{\mathrm{Z}}\left|\boldsymbol{g}_{\mathrm{Z}}\left(\mathbf{k}_{\mathrm{F}}\right)\right| / t_{\perp}\left|f_{\perp}\left(\mathbf{k}_{\mathrm{F}}\right)\right| \leq 3,
$$

favors the odd-parity PDW state. The condition may actually be satisfied in the intercalated $\mathrm{MoS}_{2}$ and $\mathrm{WS}_{2}$ as well as in the non-intercalated TMDCs with heavy metal ions and/or small carrier density. As we mentioned previously, the ratio $\alpha_{\mathrm{Z}}\left|\boldsymbol{g}_{\mathrm{Z}}\left(\mathbf{k}_{\mathrm{F}}\right)\right| / t_{\perp}\left|f_{\perp}\left(\mathbf{k}_{\mathrm{F}}\right)\right|$ can be tuned 

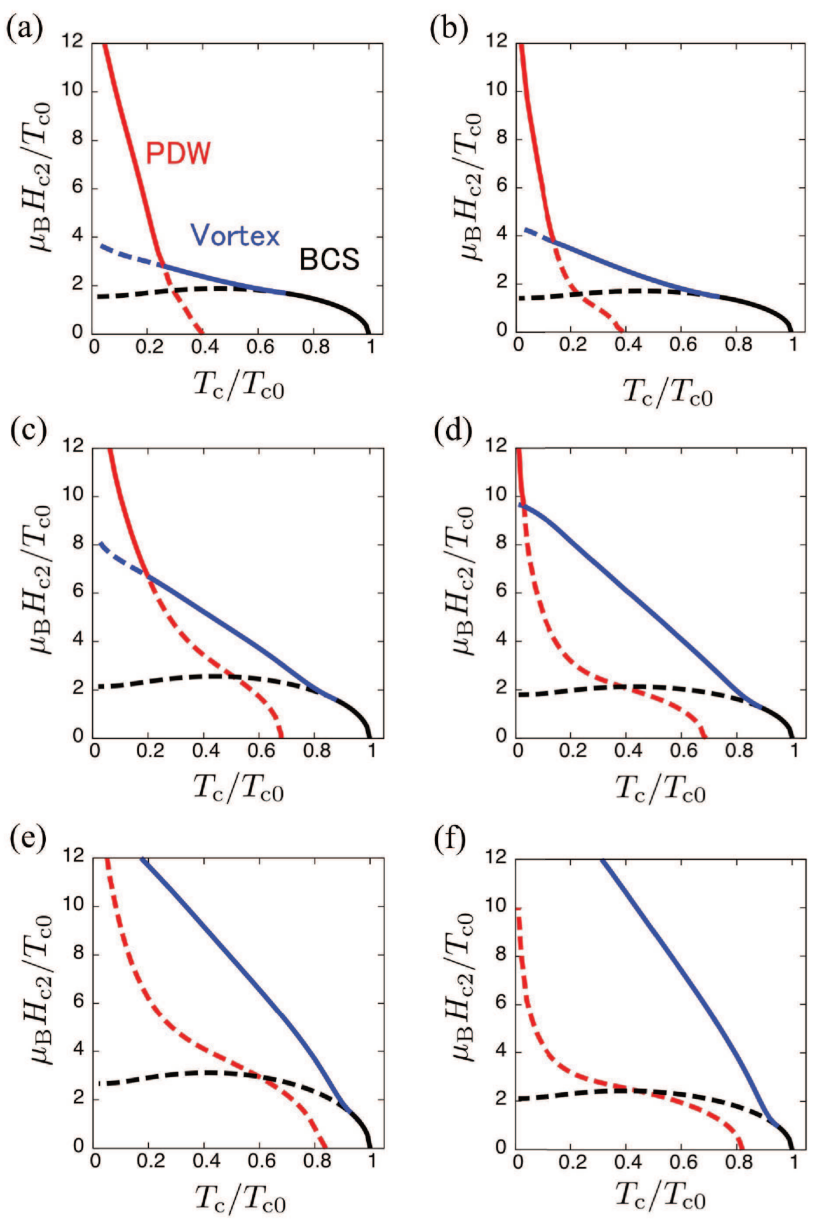

FIG. 5. (Color online) Upper critical fields of the BCS (black), PDW (red), and Josephson vortex (blue) states. The Zeemantype ASOC is chosen to be (a,b) $\alpha_{\mathrm{Z}}=0.0375$, (c,d) $\alpha_{\mathrm{Z}}=$ 0.065 , and $(\mathrm{e}, \mathrm{f}) \alpha_{\mathrm{Z}}=0.095$, in agreement with $\mathrm{MoS}_{2}, \mathrm{WS}_{2}$, and $\mathrm{WSe}_{2}$, respectively. The other parameters are the same as Fig. 2(b). The highest (realizable) upper critical field is shown by the solid line, while the fictitious ones are drawn by the dashed lines. The $c$-axis lattice constant is set to $(\mathrm{a}, \mathrm{c}, \mathrm{e}) c=$ $6.15 \AA$ for non-intercalated TMDCs and (b,d,f) $c=9.225 \AA$ for intercalated TMDCs, respectively.

by the carrier density, for which the electrostatic control has been demonstrated in various TMDCs $11,17,20-22$.

At the end of this section, we discuss the effect of increased interlayer distance by intercalation. The superconductivity has been observed in intercalated $\mathrm{K}_{x} \mathrm{MoS}_{2}$, $\mathrm{Rb}_{x} \mathrm{MoS}_{2}$, and $\mathrm{Cs}_{x} \mathrm{MoS}_{2}$ which maintain the hexagonal crystal structure 67 . The $c$-axis lattice constant increases by $35 \%-60 \%$. The two effects by intercalation have been taken into account: the interlayer hopping integral $t_{\perp}$ is decreased, and the orbital effect is enhanced. We illustrate here the enhanced orbital effect by Fig. 6. which shows the lattice constant dependence of the upper critical fields for a fixed $t_{\perp}$. It is revealed that the PDW state (Josephson vortex state) is suppressed (slightly en-
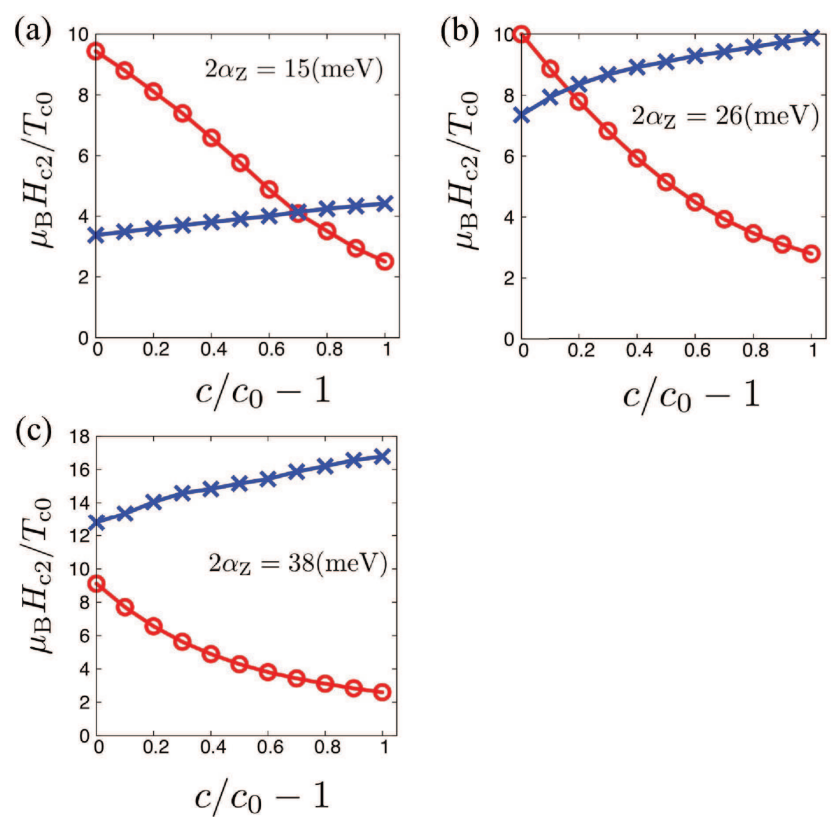

FIG. 6. (Color online) Upper critical fields of the PDW state (red circles) and Josephson vortex state (blue crosses) at $T / T_{\mathrm{c} 0}=0.1$ as a function of the interlayer distance normalized by $c_{0}=6.15 \AA$ of non-intercalated $\mathrm{MoS}_{2}$. (a) $\alpha_{\mathrm{Z}}=0.0375$, (b) $\alpha_{\mathrm{Z}}=0.065$, and (c) $\alpha_{\mathrm{Z}}=0.095$. The other parameters are the same as Fig. 5

hanced) by the orbital effect as the interlayer distance is increased. However, the PDW state is still stable for the parameters, $\alpha_{\mathrm{Z}}=0.0375$ and $c=9.225 \AA$, compatible with the intercalated $\mathrm{MoS}_{2}$.

\section{RASHBA SPIN-ORBIT COUPLING}

Up to now we have ignored the Rashba-type ASOC, because it is negligible in $\mathrm{MoS}_{2}$. Even in a strong external electric field the Rashba-type ASOC is less than $2 \%$ of the Zeeman-type ASOC17. Such a small Rashba term does not alter the superconducting phase diagram. However, we discuss here an alternative way to realize the odd-parity PDW state using a large Rashba-type ASOC, considering the tunability of spin-orbit coupling by heterostructure engineering.

Figure 7 shows the phase diagram for the Rashba-type ASOC, $\alpha_{\mathrm{R}}= \pm 0.185$. Then, the Rashba-type ASOC is comparable to the Zeeman-type ASOC on the Fermi surface, $\left|\alpha_{\mathrm{R}} \boldsymbol{g}_{\mathrm{R}}\left(\mathbf{k}_{\mathrm{F}}\right)\right| \simeq\left|\alpha_{\mathrm{Z}} \boldsymbol{g}_{\mathrm{Z}}\left(\mathbf{k}_{\mathrm{F}}\right)\right|$, for $\alpha_{\mathrm{Z}}=0.0375$. The phase diagram in the Pauli limit [Fig. [7(a)] does not show the stable PDW state, in contrast to Fig. 2(b), which shares the other parameters. Thus, the Rashbatype ASOC suppresses the PDW state in the Pauli limit. Instead, the CS state with finite Cooper pairs' momentum (see Table II) is stable in the high magnetic field region. The obtained CS state is essentially the same as 

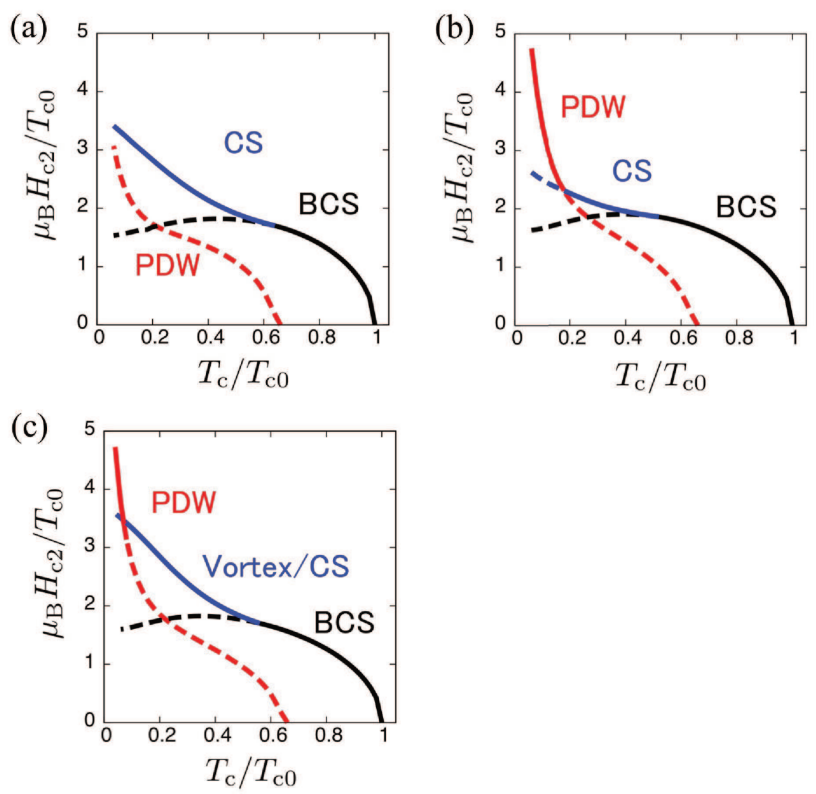

FIG. 7. (Color online) Upper critical fields of the BCS (black), PDW (red), and Josephson vortex/CS (blue) states in the presence of the Rashba-type ASOC. (a) $\alpha_{\mathrm{R}}= \pm 0.185$ and the orbital effect is neglected (Pauli limit). Taking the orbital effect into account, we obtain (b) for $\alpha_{\mathrm{R}}=+0.185$ and (c) for $\alpha_{\mathrm{R}}=-0.185$. The other parameters are $\alpha_{\mathrm{Z}}=0.0375$, $t_{\perp} / t_{1}=0.125$, and $c=6.15 \AA$.

that obtained in the bilayer Rashba mode 87 .

On the other hand, the combination of the Rashbatype ASOC and the orbital effect stabilizes the PDW state. The direction of the Cooper pairs' center-of-mass momentum $\mathbf{q}$ in the CS state is determined by the sign of the Rashba-type ASOC. In our model, the $\mathbf{q}$ is opposite between the CS state and the Josephson vortex state when $\alpha_{\mathrm{R}}>0$. Then, the effects of Rashba-type ASOC and the orbital effect are canceled, and therefore, the PDW state is stable [Fig. [7(b)], as at $\alpha_{\mathrm{R}}=0$ in the Pauli limit. Even in the opposite case, $\alpha_{\mathrm{R}}<0$, the PDW state is stable in a small parameter range [Fig. 7(c)]. These results are qualitatively consistent with what we observed in the bilayer Rashba model ${ }^{63}$.

\section{SUMMARY AND DISCUSSION}

In this work, we show the odd-parity superconductivity in bilayer TMDCs with $2 \mathrm{H}_{b}$ stacking structure. Under the parallel magnetic field, the Zeeman-type ASOC arising from the intrinsic local inversion symmetry breaking realizes the $\pi$-junction of the spin-singlet $s$-wave order parameter between two atomic layers. Such a nonuniform superconducting state in the atomic scale is called the PDW state ${ }^{58}$. The sign changing order parameter belongs to the $A_{2 u}$ irreducible representation of the $D_{3 d}$ point group. Thus, the parity of superconductivity is odd. Although the odd-parity superconductivity has attracted great interests for more than four decades, only a few spin-triplet superconductors such as $\mathrm{Sr}_{2} \mathrm{RuO}_{4}{ }^{89}$, $\mathrm{UPt}_{3}{ }^{90}$, and ferromagnetic superconductors ${ }^{91,92}$ have been identified as candidate materials. The conditions favoring the spin-triplet Cooper pairing are hardly satisfied in natural materials. Our finding shows a new and realizable mechanism of odd-parity superconductivity by means of the symmetry control enabled by the van der Waals heterostructure. Tuning the conventional $s$-wave superconductors by the spin-orbit coupling makes the odd-parity superconductivity.

The bilayer $2 \mathrm{H}_{b}$-TMDCs are promising platform of the odd-parity superconductivity, because two important conditions are naturally satisfied. First, the magnetic field required to stabilize the PDW state is parallel to the 2D plane, and therefore, the orbital effect suppressing the superconductivity is substantially avoided. Second, the interlayer hybridization vanishes at the $K$ point in the Brillouin zone owing to the quantum interference. Then, the Zeeman-type ASOC overcomes the interlayer hybridization which suppresses the PDW state. Calculating the superconducting phase diagram by taking into account both paramagnetic and orbital effects, we conclude that the intercalated bilayer $\mathrm{MoS}_{2}$ and $\mathrm{WS}_{2}$ are a platform for the odd-parity superconductivity. Recently, superconductivity has been realized in the bilayer $\mathrm{MoS}_{2}$ by gating 22 .

Finally, we discuss some properties of the PDW state to be examined by future experiments. (1) The superconducting gap in the density of states is increased 58 . (2) The spin susceptibility is enhanced $\stackrel{45}{ }$. (3) The vortex core radius shrinks 60 . (4) The zero energy Andreev bound states appear at vortex cores in the tilted magnetic field $\underline{\underline{60}}$. (5) The upper critical field exceeds the Pauli-Chandrasekhar-Clogston limit and shows upward curvature at low temperatures ${ }^{58}$. The features of the upper critical field have been universally observed in the intercalated hexagonal bulk $\operatorname{MoS}_{2} \frac{67,93}{}$, indicating a signature of the spin-orbit coupling in the superconducting state. In contrast, the upper critical field of non-hexagonal TMDCs does not exceed the PauliChandrasekhar-Clogston limit 93 . This systematic behavior points to our view on the importance of the $2 \mathrm{H}_{b}$ crystal structure.

The features (1) and (2) can be tested by thermodynamic or spectroscopic experiments, and (3) and (4) may be detected by measurements in real space, such as scanning tunneling microscopy (STM) and nuclear magnetic resonance (NMR). Although these experiments may be challenging, developments in the technology of artificial 2 D electron systems may clarify the exotic superconducting properties. The realization and identification of oddparity superconductivity in a controllable way would be a great development in the research field of superconductivity with exotic symmetry and topology. 


\section{ACKNOWLEDGEMENTS}

The authors are grateful to R. Akashi, Y. Iwasa, K. T. Law, T. Nojima, Y. Saito, T. Yoshida, N. F. Q. Yuan, and T. Watanabe for fruitful discussions. Y. N. is supported by a JSPS Fellowship for Young Scientists. This work was supported by Grant-in Aid for Scientific Research on Innovative Areas "J-Physics" (Grant No. JP15H05884) and "Topological Materials Science" (Grant No. JP16H00991) from JSPS of Japan, and by JSPS KAKENHI Grant Numbers JP15K05164 and JP15H05745.
* yanase@scphys.kyoto-u.ac.jp

1 K. F. Mak, C. Lee, J. Hone, J. Shan, and T. F. Heinz, Atomically Thin $\mathrm{MoS}_{2}$ : A New Direct-Gap Semiconductor, Phys. Rev. Lett. 105, 136805 (2010).

2 B. Radisavljevic, A. Radenovic, J. Brivio, V. Giacometti, and A. Kis, "Single-layer $\mathrm{MoS}_{2}$ transistors, Nat. Nanotechnol. 6, 147 (2011).

${ }^{3}$ Q. H. Wang, K. Kalantar-Zadeh, A. Kis, J. N. Coleman, and M. S. Strano, "Electronics and optoelectronics of twodimensional transition metal dichalcogenides, Nat. Nanotechnol. 7, 699 (2012).

4 T. Cao, G. Wang, W. Han, H. Ye, C. Zhu, J. Shi, Q. Niu, P. Tan, E. Wang, B. Liu and J. Feng, Valley-selective circular dichroism of monolayer molybdenum disulphide, Nat. Commun. 3, 887 (2012).

${ }^{5}$ K. F. Mak, K. He, J. Shan, and T. F. Heinz, Control of valley polarization in monolayer $\mathrm{MoS}_{2}$ by optical helicity, Nat. Nanotechnol. 7, 494 (2012).

${ }^{6}$ H. Zeng, J. Dai, W. Yao, D. Xiao, and X. Cui, Valley polarization in $\mathrm{MoS}_{2}$ monolayers by optical pumping, Nat. Nanotechnol. 7, 490 (2012).

7 Y. J. Zhang, T. Oka, R. Suzuki, J. T. Ye, and Y. Iwasa, "Electrically switchable chiral light-emitting transistor, Science 344, 725 (2014).

8 W. Wu, L. Wang, Y. Li, F. Zhang, L. Lin, S. Niu, D. Charnet, X. Zhang, Y. Hao, T. F. Heinz, J. Hone, and Z. L. Wang, "Piezoelectricity of single-atomic-layer $\mathrm{MoS}_{2}$ for energy conversion and piezotronics, Nature 514, 470 (2014).

9 S. Wu, S. Buckley, J. R. Schaibley, L. Feng, J. Yan, D. G. Mandrus, F. Hatami, W. Yao, J. Vučković, A. Majumder, and $\mathrm{X} . \mathrm{Xu}$, "Monolayer semiconductor nanocavity lasers with ultralow thresholds, Nature 520, 69 (2015).

10 V. K. Sangwan, D. Jariwala, I. S. Kim, K-S. Chen, T. J. Marks, L. J. Lauhon, and M. C. Hersum, "Gate-tunable memristive phenomena mediated by grain boundaries in single-layer $\mathrm{MoS}_{2}$, Nat. Nanotechnol. 10, 403 (2015).

11 J. T. Ye, Y. J. Zhang, R. Akashi, M. S. Bahramy, R. Arita, and Y. Iwasa, "Superconducting dome in a gatetuned band insulator, Science 338, 1193 (2012).

12 S. Wu, J. S. Ross, G-B. Liu, G. Aivazian, A. Jones, Z. Fei, W. Zhu, D. Xiao, W. Yao, D. Cobden, and X. Xu, Electrical tuning of valley magnetic moment through symmetry control in bilayer $\mathrm{MoS}_{2}$, Nat. Phys. 9, 149 (2013).

13 Z. Gong, G-B. Liu, H. Yu, D. Xiao, X. Cui, X. Xu, and W. Yao, Magnetoelectric effects and valley-controlled spin quantum gates in transition metal dichalcogenide bilayers, Nat. Commun. 4, 2053 (2013).

14 Y. Song, and H. Dery, Transport theory of monolayer transition-metal dichalcogenides through symmetry, Phys. Rev. Lett. 111, 026601 (2013).
15 K. F. Mak, K. L. McGill, J. Park, and E. U. McEuen, The valley Hall effect in $\mathrm{MoS}_{2}$ transistors, Science 344, 1489 (2014).

16 X. Cui, G-H. Lee, Y. D. Kim, G. Arefe, P. Y. Huang, CH.Lee, D. A. Chanet, X. Zhang, L. Wang, F. Ye, F. Piezzocchero, B. S. Jassen, K. Watanabe, T. Taniguchi, D. A. Muller, T. Low, P. Kim, and J. Hone, Multi-terminal transport measurements of $\mathrm{MoS}_{2}$ using a van der Waals heterostructure device platform, Nat. Nanotechnol. 10, 534 (2015).

17 Y. Saito, Y. Nakamura, M. S. Bahramy, Y. Kohama, J. T. Ye, Y. Kasahara, Y. Nakagawa, M. Onga, M. Tokunaga, T. Nojima, Y. Yanase, and Y. Iwasa, Superconductivity protected by spin-valley locking in ion-gated $\mathrm{MoS}_{2}$, Nat. Phys. 12, 144 (2016).

18 J. M. Lu, O. Zeliuk, I. Leemakers, Naoh F. Q. Yuan, U. Zeitler, K. T. Law, and J. T. Ye, Evidence for twodimensional Ising superconductivity in gated $\mathrm{MoS}_{2}$, Science 350, 1353 (2015).

19 X. Xi, Z. Wang, W. Zhao, J. H. Park, K. T. Low, H. Berger, L, Folló, J. Shan, and K. F. Mak, Ising pairing in superconducting $\mathrm{NbSe}_{2}$ atomic layers, Nat. Phys. 12, 139 (2015).

${ }^{20}$ W. Shi, J. Ye, Y. Zhang, R. Suzuki, M. Yoshida, J. Miyazaki, N. Inoue, Y. Saito, and Y. Iwasa, Superconductivity series in transition metal dichalcogenides by ionic gating, Sci. Rep. 5, 12534 (2015).

21 S. Jo, D. Costanzo, H. Berger, and A. F. Morpurgo, Electrostatically induced superconductivity at the surface of $\mathrm{WS}_{2}$, Nano Lett. 15, 1197 (2015).

22 D. Costanzo, S. Jo, H. Berger, and A. F. Morpurgo, Gateinduced superconductivity in atomically thin $\mathrm{MoS}_{2}$ crystals, Nat. Nanotechnol. 11, 339 (2016).

23 J. M. Lu, O. Zheliuk, Q. H. Chen, I. Leermakers, N. E. Hussey, U. Zeitler, and J. T. Ye, A full superconducting dome of strong Ising protection in gated monolayer $\mathrm{WS}_{2}$, arXiv:1703.06369.

24 G. Liu, D. Xiao, Y. Yao, X. Xude, and W. Yao, Electronic structures and theoretical modelling of two-dimensional group-VIB transition metal dichalcogenides, Chem. Soc. Rev. 44, 2643 (2015).

25 D. Xiao, G. B. Liu, W. Feng, X. Xu, and W. Yao, Coupled spin and valley physics in monolayers of $\mathrm{MoS}_{2}$ and other group-VI dichalcogenides, Phys. Rev. Lett. 108, 196802 (2012).

26 Z. Y. Zhu, Y. C. Cheng, and U. Schwingenschlögl, Giant spin-orbit-induced spin splitting in two-dimensional transition-metal dichalcogenide semiconductors, Phys. Rev. B 84, 153402 (2011).

27 T. Cheiwchanchamnangij and W. R. L. Lambrechet, Quasiparticle band structure calculation of monolayer, bilayer, and bulk MoS 2 , Phys. Rev. B 85, 205302 (2012). 
28 E. S. Kadantsev and P. Hawrylak, Electronic structure of a single $\mathrm{MoS}_{2}$ monolayer, Solid State Commun. 152, 909 (2012).

${ }^{29}$ K. Kośmider, J. W. González, and J. F. Fernández-Rossier, Large spin splitting in the conduction band of transition metal dichalcogenide monolayers, Phys. Rev. B 88, 245436 (2013).

30 A. Kormányos, G. Burkard, M. Gmitra, J. Fabian, V.Zályomi, N. D. Drummond, and V. Fal'ko, k·p theory for two-dimensional transition metal dichalcogenide semiconductors, 2D Mater. 2, 022001 (2015).

31 R. Coehoorn, C. Hass, J. Dijkstra, and C. J. F. Flipse, Electronic structure of $\mathrm{MoSe}_{2}, \mathrm{MoS}_{2}$, and $\mathrm{WSe}_{2}$. I. Bandstructure calculations and photoelectron spectroscopy, Phys. Rev. B 35, 6195 (1987).

32 C. Autieri, A. Bouhon, and B. Sanyal, Gap opening and large spin-orbit splitting in $\mathrm{MX}_{2}(\mathrm{M}=\mathrm{Mo}, \mathrm{W} \mathrm{X}=\mathrm{S}, \mathrm{Se}, \mathrm{Te})$ from the interplay between crystal field and hybridizations: insights from ab-initio theory, arXiv:1602.02625.

33 B. S. Chandrasekhar, A note on the maximum critical field of high-field superconductors, Appl. Phys. Lett. 1, 7 (1962).

34 A. M. Clogston, Upper limit for the critical field in hard superconductors, Phys. Rev. Lett. 9, 266 (1962).

35 A. M. Sánchez, D. Sangalli, K. Hummer, A. Marini, and W. Wirtz, Effect of spin-orbit interaction on the optical spectra of single-layer, double-layer, and bulk $\mathrm{MoS}_{2}$, Phys. Rev. B 88, 045412 (2013).

36 N. F. Q. Yuan, K. F. Mak, and K. T. Law, Possible Topological Superconducting Phases of $\mathrm{MoS}_{2}$, Phys. Rev. Lett. 113, 097001 (2014).

37 W. He, B. T. Zhou, J. J. He, T. Zhang, and K. T. Law, Nodal Topological Superconductivity in Monolayer $\mathrm{NbSe}_{2}$, arXiv:1604.02867.

38 Y-T. Hsu, A. Vaezi, M. H Fischer, and E.-A. Kim, Topological superconductivity in monolayer transition metal dichalcogenides, Nat. Commun. 8, 14985 (2017).

39 B. T. Zhou, N. F. Q. Yuan, H. Jiang, and K. T. Law, Ising superconductivity and Majorana fermions in transitionmetal dichalcogenides, Phys. Rev. B 93, 180501(R) (2016).

40 J. Zhang and V. Aji, Topological Yu-Shiba-Rusinov chain in monolayer transition-metal dichalcogenide superconductors, Phys. Rev. B 94, 060501 (2016).

41 G. Sharma and S. Tewari, Yu-Shiba-Rusinov states and topological superconductivity in Ising paired superconductors, Phys. Rev. B 94, 094515 (2016).

${ }^{42}$ R. Wakatsuki and K. T. Law, Proximity effect and Ising superconductivity in superconductor/transition metal dichalcogenide heterostructures, arXiv:1604.04898.

43 M. A. Cazalilla, H. Ochoa, and F. Guinea, Quantum spin hall effect in two-dimensional crystals of transition-metal dichalcogenides, Phys. Rev. Lett. 113, 077201 (2014).

44 J. A. Wilson and A. D. Yoffe, The transition metal dichalcogenides discussion and interpretation of the observed optical, electrical and structural properties, Adv. Phys. 18, 193 (1969).

45 D. Maruyama, M. Sigrist, and Y. Yanase, Locally Noncentrosymmetric Superconductivity in Multilayer Systems, J. Phys. Soc. Jpn. 81, 034702 (2012).

46 M. H. Fischer, F. Loder, and M. Sigrist, Superconductivity and local noncentrosymmetry in crystal lattices, Phys. Rev. B 84, 184533 (2011).

47 S. K. Goh, Y. Mizukami, H. Shishido, D. Watanabe, S. Yasumoto, M. Shimozawa, M. Yamashita, T. Terashima,
Y. Yanase, T. Shibauchi, A. I. Buzdin, and Y. Matsuda, Anomalous Upper Critical Field in $\mathrm{CeCoIn}_{5} / \mathrm{YbCoIn}_{5} \mathrm{Su}^{-}$ perlattices with a Rashba-Type Heavy Fermion Interface, Phys. Rev. Lett. 109, 157006 (2012).

${ }^{48}$ M. Shimozawa, S. K. Goh, R.kobayashi, T. Watashige, Y. Mizukami, H. Ikeda, H. Shishido, Y. Yanase, T. Terashima, T. shibauchi, and Y. Matsuda, Controllable Rashba spinorbit Interaction in artificially engineered superlattices involving the heavy-fermion superconductor $\mathrm{CeCoIn}_{5}$, Phys. Rev. Lett. 112, 156404 (2014).

49 X. Zhang, Q. Liu, J. Luo, A. J. Freeman, and A. Zunger, Hidden spin polarization in inversion-symmetric bulk crystals, Nat. Phys. 10, 387 (2014).

50 J. M. Riley, F. Mazzola, M. Dendzik, M. Michiardi, T. Takayama, L. Bawden, C. Granerod, M. Leandersson, T. Balasubramanian, M. Hoesch, T. K. Kim, H. Takagi, W. Meevasana, Ph. Hofmann, M. S. Bahramy, J. W. Wells, and P. D. C. King, Direct observation of spin-polarized bulk bands in an inversion-symmetric semiconductor, Nat. Phys. 10, 835 (2014).

51 A. M. Jones, H. Yu, J. S. Ross, P. Klement, N. J. Ghimire, J. Yan, D. G. Mandrus, W. Yao, and X. Xu, Spin-layer locking effects in optical orientation of exciton spin in bilayer $\mathrm{WSe}_{2}$, Nat. Phys. 10, 130 (2014).

52 M. Gehlmann, G. Bihlmayer, I. Aguilera, E. Mlynczak, M. Eschbach, S. Doring, P. Gospodaric, S. Cramm, B. Kardynal, L. Plucinski, S. Blugel, and C. M. Schneider, Quasi 2D electronic states with high spin-polarization in centrosymmetric $\mathrm{MoS}_{2}$ bulk crystals, Sci. Rep. 6, 26197 (2016).

53 D. Santos-Cottin, M. Casula, G. Lantz, Y. Klein, L. Petaccia, P. Le Fevre, F. Bertran, E. Papalazarou, M. Marsi, and A. Gauzzi, Rashba coupling amplification by a staggered crystal field, Nat. Commun. 7, 11258 (2016).

54 Non-centrosymmetric Superconductors: Introduction and Overview, Lecture Notes in Physics Vol. 847, edited by E. Bauer and M. Sigrist (Springer-Verlag, Berlin, Heidelberg, 2012).

55 M. Smidman, M. B. Salamon, H. Q. Yuan, and D. F. Agterberg, Superconductivity and spin-orbit coupling in noncentrosymmetric materials: a review, Rep. Prog. Phys. 80, 036501 (2017).

${ }^{56}$ L. Fu and E. Berg, Odd-Parity Topological Superconductors: Theory and Application to $\mathrm{Cu}_{x} \mathrm{Bi}_{2} \mathrm{Se}_{3}$, Phys. Rev. Lett. 105, 097001 (2010).

57 S. Nakosai, Y. Tanaka, and N. Nagaosa, Topological Superconductivity in Bilayer Rashba System, Phys. Rev. Lett. 108, 147003 (2012).

${ }^{58}$ T. Yoshida, M. Sigrist, and Y. Yanase, Pair-density wave states through spin-orbit coupling in multilayer superconductors, Phys. Rev. B 86, 134514 (2012).

59 T. Yoshida, M. Sigrist, and Y. Yanase, Parity-mixed Superconductivity in Locally Non-centrosymmetric System, J. Phys. Soc. Jpn. 83, 013703 (2014).

60 Y. Higashi, Y.Nagai, T. Yoshida Y, Masaki, and Y. Yanase, Robust zero-energy bound states around a pair-densitywave vortex core in locally noncentrosymmetric superconductors, Phys. Rev. B 93, 104529 (2016).

61 S. Sumita and Y. Yanase, Superconductivity in magnetic multipole states, Phys. Rev. B 93, 224507 (2016).

62 T. Yoshida, M. Sigrist, and Y. Yanase, Topological Crystalline Superconductivity in Locally Noncentrosymmetric Multilayer Superconductors, Phys. Rev. Lett. 115, 027001 (2015). 
63 T. Watanabe, T. Yoshida, and Y. Yanase, Odd-parity superconductivity by competing spin-orbit coupling and orbital effect in artificial heterostructures, Phys. Rev. B 92, 174502 (2015).

64 Tsuneya Yoshida, A. Daido, Y. Yanase, and N. Kawakami, Fate of Majorana modes in $\mathrm{CeCoIn}_{5} / \mathrm{YbCoIn}_{5}$ superlattices - a test bed for reduction of topological classification-, Phys. Rev. Lett. 118, 147001 (2017).

65 R. Akashi, M. Ochi, R. Suzuki, S. Bordács, Y. Tokura, Y. Iwasa, and R. Arita, Two-dimensional valley electrons and excitons in noncentrosymmetric $3 \mathrm{R}-\mathrm{MoS}_{2}$, Phys. Rev. Applied 4, 014002 (2015).

66 R. Akashi, Y. Iida, K. Yamamoto, K. Yoshizawa Interference of the Bloch phase and stacking geometry in layered materials, Phys. Rev. B 95, 245401 (2017).

67 J. A. Woollam, and R. B. Somoano, Physics and Chemistry of $\mathrm{MoS}_{2}$ Intercalation Compounds, Materials Science and Engineering 31, 289 (1977).

${ }^{68}$ P. Fulde and R. A. Ferrell, Superconductivity in a Strong Spin-Exchange Field, Phys. Rev. 135, A550 (1964).

69 A. I. Larkin and Y. N. Ovchinnikov, Zh. Eksp. Teor. Fiz. 47, 1136 (1964); Inhomogeneous State of Superconductors, Sov. Phys. JETP. 20762 (1965).

70 A. I. Buzdin, Proximity effects in superconductorferromagnet heterostructures, Rev. Mod. Phys. 77, 935 (2005).

71 Y. Matsuda and H. Shimahara, Fulde-Ferrell-LarkinOvchinnikov State in Heavy Fermion Superconductors, J. Phys. Soc. Jpn. 76, 051005 (2007).

72 T. Brumme, M. Calandra, and F. Mauri, First-principles theory of field-effect doping in transition-metal dichalcogenides: Structural properties, electronic structure, Hall coefficient, and electrical conductivity, Phys. Rev. B 91, 155436 (2015).

73 S. Fang, R. K. Defo, S. Shirodkar, S. Lieu, G. Tritsaris, and E. Kaxiras, Ab initio tight-binding Hamiltonian for transition metal dichalcogenides, Phys. Rev. B 92, 205108 (2015).

74 N. Podberezskaya, S. Magarill, N. Pervukhina, and S. Borisov, Crystal Chemistry of Dichalcogenides $\mathrm{MX}_{2}, \mathrm{~J}$. Struct. Chem. 42, 654 (2001).

75 G-B. Liu, W-Y. Shan, Y. Yao, W. Yao, and D. Xiao, Three-band tight-binding model for monolayers of groupVIB transition metal dichalcogenides, Phys. Rev. B 88, 085433 (2013).

${ }^{76}$ L. F. Mattheiss, Band Structures of Transition-MetalDichalcogenide Layer Compounds, Phys. Rev. B 8, 3719 (1973).

77 P. A. Frigeri, Superconductivity in Crystals Without an Inversion Center, PhD thesis, ETH Zurich (2005).
78 W. J. Schutte, J. L. de Boer, and F. Jellinek, Crystal structures of Tungsten Disulfide and Diselenide, J. Solid State Chem. 70, 207 (1987).

79 T. Böker, R. Severin, A. Müller, C. Janowitz, R. Manzke, D. Vob, P. Krügar, A. Mazur, and J. Pollmann, Band structure of $\mathrm{MoS}_{2}, \mathrm{MoSe}_{2}$, and $\alpha-\mathrm{MoTe}_{2}$ : Angle-resolved photoelectron spectroscopy and ab initio calculations, Phys. Rev. B 64, 235305 (2001).

80 A. Ramasubramaniam, D. Naveh, and E. Towe, Tunable band gaps in bilayer transition-metal dichalcogenides, Phys. Rev. B 84, 205325 (2011).

81 R. Roldán, E. Cappelluti, and F. Guinea, Interactions and superconductivity in heavily doped $\mathrm{MoS}_{2}$, Phys. Rev. B 88, 054515 (2013).

82 J. Yuan and C. Honerkamp, Triplet pairing driven by Hund's coupling in doped monolayer $\mathrm{MoS}_{2}$, arXiv:1504.04536.

83 Y. Ge and A. Y. Liu, Phonon-mediated superconductivity in electron-doped single-layer $\mathrm{MoS}_{2}$ : A first-principles prediction, Phys. Rev. B 87, 241408(R) (2013).

84 M. Rösner, S. Haas, and T. O. Wehling, Phase diagram of electron-doped dichalcogenides, Phys. Rev. B 90, 245105 (2014).

85 T. Das and K. Dolui, Superconducting dome in $\mathrm{MoS}_{2}$ and $\mathrm{TiSe}_{2}$ generated by quasiparticle-phonon coupling, Phys. Rev. B 91, 094510 (2015).

86 D. F. Agterberg, M. Sigrist, and H. Tsunetsugu, Phys. Rev. Lett. 102, 207004 (2009).

87 T. Yoshida, M. Sigrist, and Y. Yanase, Complex-Stripe Phases Induced by Staggered Rashba Spin-Orbit Coupling, J. Phys. Soc. Jpn. 82, 074714 (2013).

88 C.-X. Liu, Unconventional superconductivity in bilayer transition metal dichalcogenides, Phys. Rev. Lett. 118, 087001 (2017).

89 Y. Maeno, S. Kittaka, T. Nomura, S. Yonezawa, and K. Ishida, Evaluation of spin-triplet superconductivity in $\mathrm{Sr}_{2} \mathrm{RuO}_{4}$, J. Phys. Soc. Jpn. 81, 011009 (2012).

90 R. Joynt and L. Taillefer, The superconducting phases of $\mathrm{UPt}_{3}$, Rev. Mod. Phys. 74, 235 (2002).

91 S. S. Saxena, P. Agarwal, K. Ahilan, F. M. Grosche, R. K. W. Haselwimmer, M. J. Steiner, E. Pugh, I. R. Walker, S. R. Julian, P. Monthoux, G. G. Lonzarich, A. Huxley, I. Sheikin, D. Braithwaite and J. Flouquet, Superconductivity on the border of itinerant-electron ferromagnetism in $\mathrm{UGe}_{2}$, Nature (London) 406, 587 (2000).

92 D. Aoki and J. Flouquet, Ferromagnetism and superconductivity in uranium compounds, J. Phys. Soc. Jpn. 81, 011003 (2012).

93 J. A. Woollam, and R. B. Somoano, Superconducting critical fields of alkali and alkaline-earth intercalates of $\mathrm{MoS}_{2}$, Phys. Rev. B 13, 3843 (1976). 